\title{
Amyloidogenicity of regions of Nucleophosmin 1: A direct link between protein misfolding and Acute Myeloid Leukemia
}

\author{
Daniela Marasco, Ettore Novellino, Giancarlo Morelli, Sara La Manna
}

University of Naples Federico II, Italy

https://doi.org/10.17952/35EPS.2018.038

Nucleophosmin (NPM1) is a multidomain protein involved in a variety of biological processes and identified as the most frequently mutated gene in Acute Myeloid Leukemia (AML). Its C-terminal domain (CTD) is endowed with a three helix bundle tertiary structure: H1 (243-259), H2 (264-277) and H3 (280-294) helices fold through a compact transition state and unfold keeping a residual secondary structure, they are held together by an aromatic core at the interface between $\mathrm{H} 2$ and $\mathrm{H} 3$ helices [1]. AML mutations cause the loss of tryptophans 288 and 290 in the third helix of the CTD, that, in the wt form constitute a NOLS (nucleolar localization signal), and the creation of an additional leucine-rich nuclear export signal (NES), inducing an aberrant cytoplasmic dislocation (named NPM1c+). AML cells expressing NPM1c+ present differentiation arrest, apoptosis blockage, impaired DNA repair, and induced cell proliferation. Many important tumor suppressors are deregulated either directly or indirectly by NPM1c+ namely- p14ARF, p53, PTEN and also caspases-6 and -8 resulted inhibited with altered apoptotic pathways [2]. There are many different strategies in targeting NPM1 such as i) NPM1 oligomerization, ii) its interctome, iii) its post-translational modifications, iv) its selective displacement from nucleoli that causes nucleolar stress and consequent apoptotic cell death, following the so-called "nucleolar starvation hypothesis" [2].

Few years ago, through a protein dissection approach we started to analyse the structural and functional independence of each helix of the CTD. The first study revealed that the first helix holded for an uncommon helical content intrinsically endowed with an unusual thermal stability [3]. More surprisingly the neighbouring regions $\mathrm{H} 2$ [4] [5] and $\mathrm{H} 3$ in AML mutated variants [6] separately revealed able to form amyloid-like assemblies endowed with fibrillar morphology and $\beta$-sheet structure. These aggregates proved to be toxic in cell viability assays.

Furthermore, a short stretch (nine residues) spanning 264-272 region, derived from the aromatic core of the bundle and located within $\mathrm{H} 2$, revealed the most amyloidogenic fragment of the entire protein. Solution and fiber state investigations through Circular Dichroism, Fluorescence spectroscopies, amyloid seeding assay (ASA), isothermal titration calorimetry (ITC) and Electrospray ionization (ESI) mass analyses, demonstrated that this peptide is able to self-aggregate and that helical conformation plays a crucial role in the aggregation mechanism. Indeed related nanostructures, investigated by means of Scanning Electron Microscopy (SEM) and Wide-Angle $\mathrm{X}$-ray Scattering (WAXS), revealed flat with twisted profiles and typical cross- $\beta$-structures [7].

Amyloidogenic CTD regions demonstrated able to interact with model systems of membranes with different mechanisms and the presence of cholesterol revealed of crucial importance. Indeed the interaction of CTDNPM1 amyloidogenic regions with lipid membranes was analysed through fluorescence, SPR, CD and ESR spectroscopies and by immune-fluorescence in leukemic cells. Resulting data indicate that $\mathrm{H} 2$ peptide showed a direct interaction with cholesterol within the membrane causing a sensitive fluidification of the bilayer. Noticeably, NPM1mutA (the most common AML mutation) thicken at the plasma membrane, differently from wt NPM1. These findings are in accordance with diverse mechanisms of interaction of wt CTD and Cterm mutA toward membrane models analyzed in vitro [8].

More recently, we found that all AML mutations, from A to F, without both or one Trp of the NOLS (nucleolar localization signal) induce amyloid-like aggregation and cytotoxicity. Using a combination of biophysical techniques including ThT-fluorescence, CR absorbance, CD, SEM (figure 1) and WAXS on a series of peptides bearing mutations we evidenced that the amyloidogenicity is directly linked to AML. The type of mutation influence the cytotoxicity in neuroblastoma cells and the morphology of amyloid fibrils. 


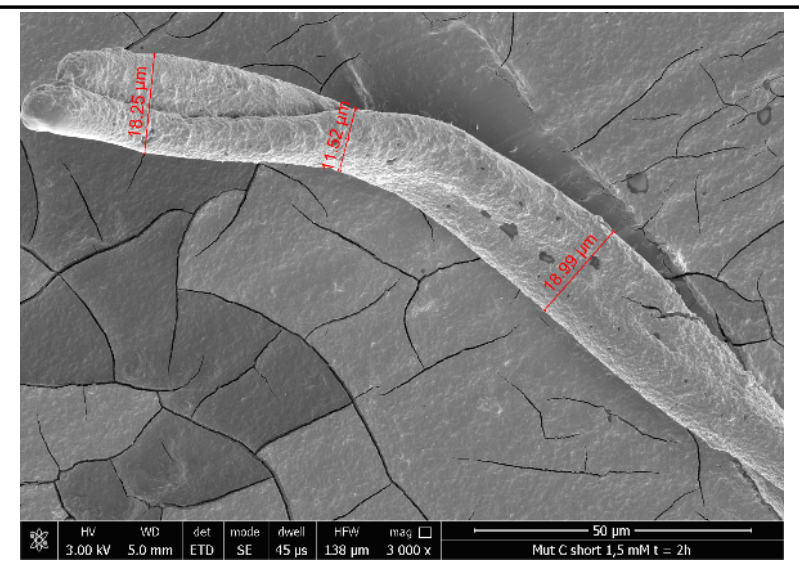

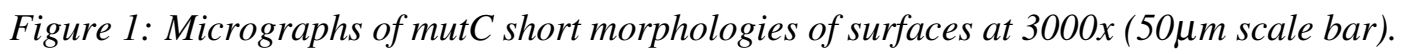

Our results demonstrate, unequivocally, that separated regions of the CTD of NPM1 are prone to aggregate to amyloid states: thus we formulated a mechanicistic hypothesis where the destabilization of the helical bundle by AML-mutations predispose it to the formation of toxic aggregates causing the exposure of the $\mathrm{H} 2$ and $\mathrm{H} 3$ regions.

Since the oligomeric state of NPM1 is of outmost importance for its biological roles in its wild type form, in the light of our results we can argue that the contribution of the CTD to the oligomeric/aggregated form could be directly linked the leukomogenic potentials of AML mutations. Departing away from the fragmentation approach for the explanation of the behaviour of the entire mutated protein, we investigated the polypeptide covering the third and second helices of the bundle of type A mutated CTD. Also this region, spanning 264-298 residues, demonstrated an amyloid aggregation resulting from a self-recognition mechanism and soluble assemblies resulted cytoxic in MTT assay corroborating a therapeutic strategy in AML consisting in the self-degradation of mutated protein [9].

Since structural studies are crucial in drug discovery process focused on NPM1c+/AML, these studies are of utmost importance in innovative strategies to identify more potent NPM1-targeted drugs.

\section{References}

[1] C.G. Grummitt, F.M. Townsley, C.M. Johnson, A.J. Warren, M. Bycroft, Structural consequences of nucleophosmin mutations in acute myeloid leukemia, The Journal of Biological Chemistry 283(34) (2008) 23326-32.

[2] A. Di Matteo, M. Franceschini, S. Chiarella, S. Rocchio, C. Travaglini-Allocatelli, L. Federici, Molecules that target nucleophosmin for cancer treatment: an update, Oncotarget (2016).

[3] P.L. Scognamiglio, C. Di Natale, M. Leone, M. Poletto, L. Vitagliano, G. Tell, D. Marasco, G-quadruplex DNA recognition by nucleophosmin: new insights from protein dissection, Biochimica et Biophysica Acta 1840(6) (2014) 2050-9.

[4] C. Di Natale, P.L. Scog namiglio, R. Cascella, C. Cecchi, A. Russo, M. Leone, A. Penco, A. Relini, L. Federici, A. Di Matteo, F. Chiti, L. Vitagliano, D. Marasco, Nucleophosmin contains amyloidogenic regions that are able to form toxic aggregates under physiological conditions, FASEB journal: official publication of the Federation of American Societies for Experimental Biology (2015).

[5] A. Russo, C. Diaferia, S. La Manna, C. Giannini, T. Sibillano, A. Accardo, G. Morelli, E. Novellino, D. Marasco, Insights into amyloid-like aggregation of $\mathrm{H} 2$ region of the $\mathrm{C}$-terminal domain of nucleophosmin, Bba-Proteins Proteom 1865(2) (2017) 176-185.

[6] P.L. Scognamiglio, C. Di Natale, M. Leone, R. Cascella, C. Cecchi, L. Lirussi, G. Antoniali, D. Riccardi, G. Morelli, G. Tell, F. Chiti, D. Marasco, Destabilisation, aggregation, toxicity and cytosolic mislocalisation of nucleophosmin regions associated with acute myeloid leukemia, Oncotarget 7(37) (2016) 59129-59143.

[7] S. La Manna, Roviello V., Scognamiglio, P. L., Diaferia, C., Giannini, C., Sibillano, T., Morelli, G., Novellino 
E., Marasco D., Amyloid fibers deriving from the aromatic core of C-terminal domain of nucleophosmin 1, International Journal of Biological Macromolecules, under review (2018).

[8] A. De Santis, S. La Manna, I.R. Krauss, A.M. Malfitano, E. Novellino, L. Federici, A. De Cola, A. Di Matteo, G. D'Errico, D. Marasco, Nucleophosmin-1 regions associated with acute myeloid leukemia interact differently with lipid membranes, Biochimica et Biophysica Acta 1862(4) (2018) 967-978.

[9] C. Di Natale, La Manna, S., Malfitano, A., Di Somma, S., Florio D., Scognamiglio, P. L., Novellino E., Netti, P. A., Marasco D., Structural and kinetic insights into amyloid aggregation of a polypeptide covering the second and the third helices of the type A mutation of Nucleophosmin 1 in Acute Myeloid Leukemia, Bioorg Chem, under review (2018). 